\title{
Beschäftigungspolitische Krisenreaktionen in Deutschland, Italien und dem Vereinigten Königreich
}

Alle großen Länder in der Europäischen Union wurden heftig von der Großen Rezession getroffen. Doch trotz massiver Einbrüche des Bruttoinlandsprodukts ist der Anstieg der Arbeitslosigkeit deutlich hinter den Befürchtungen und Erfahrungen zurückgeblieben. Dennoch sind die Länder unterschiedlich gut durch die Krise gekommen. Während man bei Deutschland vom Arbeitsmarktwunder spricht, ist das Vereinigte Königreich mit einer Arbeitslosenquote konfrontiert, wie sie das Land schon lange Zeit nicht mehr gesehen hat. Italiens Bilanz liegt zwischen der der beiden anderen Länder. Diese unterschiedlichen Entwicklungen sind teilweise auf unterschiedliche beschäftigungspolitische Strategien zurückzuführen.

\section{1 \\ Einleitung}

Nachdem 2007 spekulative Blasen im amerikanischen Häusermarkt geplatzt sind, hat sich die Immobilienkrise zu einer globalen Wirtschaftskrise entwickelt und rasch über Europa ausgebreitet. Bis Mitte 2008 waren die meisten Europäischen Länder von der Rezession betroffen (Leschke/Watt 2010, S. 5). Dabei haben sie ähnliche Erfahrungen gemacht. Die Krise war so heftig, dass in vielen Ländern das Bruttoinlandsprodukt (BIP) so stark gefallen ist wie nie zuvor. Trotz dieses starken Rückgangs ist die Arbeitslosigkeit fast nirgendwo so stark angestiegen wie es vorangegangene Erfahrungen hätten vermuten lassen, so z. B. in Deutschland, Italien und dem Vereinigten Königreich (VK). Hier ging das BIP vom Höhepunkt des letzten Aufschwungs bis zum Konjunkturtief um 6,7 \% bzw. $6 \%$ (VK) zurück (OECD 2010a). Obwohl die Arbeitslosigkeit in allen drei Fällen weniger als erwartet stieg, hat sie sich doch deutlich unterschiedlich entwickelt. Während in Deutschland die Arbeitslosigkeit im gleichen Zeitraum sogar um $0,9 \%$ gefallen ist, stieg sie in Italien moderat um $1,5 \%$ und im VK mit 2,3\% etwas stärker (OECD 2010b). Auffallend ist dabei, wie ähnlich die Krise Deutschland und Italien getroffen hat. Beide Länder gehören zu der Gruppe von Ländern, die von der Organisation für wirtschaftliche Zusammenarbeit und Entwicklung (OECD) (2010b, S. 32) folgendermaßen charakterisiert werden: Rückgang in den Exporten als Anteil vom
Bruttoinlandsprodukt von mindestens fünf Prozentpunkten, Rückgang in der Arbeitsproduktivität von mindestens fünf Prozentpunkten sowie mindestens $1 \%$ aller Beschäftigten in Kurzarbeit. Für das VK waren diese Effekte zwar nicht so stark ausgeprägt, Exporte und Arbeitsproduktivität gingen aber auch hier deutlich zurück. Kurzarbeit spielte keine bedeutende Rolle.

Diese gemeinsame Erfahrung zusammen mit den gemeinsamen Zielen - die Märkte zu stabilisieren, die Nachfrage zu stimulieren und die Beschäftigung zu sichern - macht einen Vergleich der drei Länder besonders fruchtbar. Zudem gehören sie zu den vier größten Volkswirtschaften der Europäischen Union ${ }^{1}$ mit zusammengenommen mehr als der Hälfte der Bevölkerung und knapp der Hälfte des Bruttoinlandsprodukts. Jedes dieser Länder kann als große Volkswirtschaft betrachtet werden, die Nebeneffekte auf andere (mit ihr ökonomisch verbundene) Länder verursacht. Somit kann sich auch keines dieser Länder ausschließlich auf andere Länder verlassen und warten, dass deren Krisenmaßnahmen im eigenen Land ausreichend Wirkung bei der Krisenbekämpfung zeigen. Jedes muss eigenständig Maßnahmen ergreifen, um mit den von der Krise verursachten Problemen fertig zu werden.

Die Frage ist, ob in den oben genannten Ländern klare beschäftigungspolitische Strategien zur Krisenbewältigung erkennbar sind und welche Instrumente dabei zur Verfügung standen und zum Einsatz kamen. Im Folgenden werden mit Hilfe von drei Länderstudien Antworten auf diese Frage gesucht. Dabei arbeiten wir heraus, wie weit die Länder auf gleiche Strategien und Maßnahmen zurückgegriffen haben und in welchem Umfang länderspezifische Instrumente genutzt wurden (Abschnitte 3-5). Zuvor geben wir einen kurzen Überblick über die länderspezifische Krisenbetroffenheit sowie die Wirtschafts- und Beschäftigungsstruktur (Abschnitt 2). Nach der Präsentation der Länderstudien endet der Artikel mit einer vergleichenden Diskussion (Abschnitt 6) und einem Fazit (Abschnitt 7).

\section{Länderspezifische Krisenbetroffenheit}

Um ein besseres Verständnis für die länderspezifische Krisenbewältigung zu erhalten, ist es wichtig zu analysieren, wie die einzelnen Länder durch die Krise getroffen wurden. Italien wurde zunächst nicht so

\footnotetext{
Zum Vergleich von Frankreich und Deutschland
} siehe Sturn/van Treeck in diesem Heft.

Ulrike Stein, PhD, ist Wissenschaftlerin
im Institut für Makroökonomie und
Konjunkturforschung (IMK) in der Hans-
Böckler-Stiftung.
Arbeitsschwerpunkte: Panelanalysen,
Einkommensverteilung, Arbeitsmarkt.
e-mail: Ulrike-Stein@boeckler.de
Fabio Aricò, PhD, ist Lecturer an der
University of St Andrews in Schottland.
Arbeitsschwerpunkte: Arbeitsmarkt, Bildung,
Technologischer Wandel.
e-mail: Fabio.Arico@st-andrews.ac.uk


stark wie Deutschland oder das VK von der globalen Krise getroffen, da es keine Bankenkrise hatte, sondern nur mit dem globalen Nachfrageschock konfrontiert war: vor allem im Maschinenbau, in der Textilindustrie sowie der Bau- und Automobilindustrie. Deutschland hatte sowohl eine Bankenkrise als auch einen Einbruch in der Nachfrage. Damit wurden hauptsächlich der Exportsektor, insbesondere die Investitionsgüterindustrie im Produzierenden Gewerbe, der Maschinenbau und die Automobilbranche in Mitleidenschaft gezogen. Das VK wurde von der Krise gleich durch drei verschiedene Kanäle getroffen. Zum einen wurde die britische Volkswirtschaft durch die große Abhängigkeit von der Finanzbranche (Abbildung 1) schwer von der Banken- und Finanzkrise getroffen, wodurch sowohl die Unternehmensinvestitionen als auch die Konsumausgaben der Haushalte stark eingeschränkt wurden. Zudem erlebte das VK eine Immobilienkrise. Durch den starken Fall in den Immobilienpreisen brach die Baubranche ein. Das Vereinigte Königreich erlitt aufgrund des globalen Nachfrageinbruchs direkte negative Auswirkungen auf die Exportindustrie.

Die Vermittlung der Krise durch verschiedene Kanäle in den drei Ländern hängt unter anderem mit Unterschieden in der branchenspezifischen Beschäftigungsstruktur und Wirtschaftsleistung, gemessen am branchenspezifischen Anteil am Bruttoinlandsprodukt, zusammen (Abbildung 1). Besonders auffällig sind die Unterschiede in Hinblick auf die Abhängigkeit vom Bankensektor und das Verarbeitende Gewerbe. Während das VK mit Abstand die höchste Abhängigkeit von der Finanzbranche hat, ist in Italien und Deutschland die Bedeutung des Produzierenden Gewerbes erkennbar. In Deutschland sticht außerdem besonders der geringe Anteil der Branchen Handel und Dienstleistungssektor am Bruttoinlandsprodukt hervor, insbesondere da immerhin $45 \%$ der Beschäftigten in diesen beiden Branchen arbeiten.

\section{Deutschland}

\subsection{VOR DER KRISE}

Die deutsche Wirtschaft ist durch eine starke Exportorientierung geprägt. Auf-

\section{Abb. 1: Bruttoinlandsprodukt (BIP) und Beschäftigte (BE) nach \\ Branchen - in \% -}

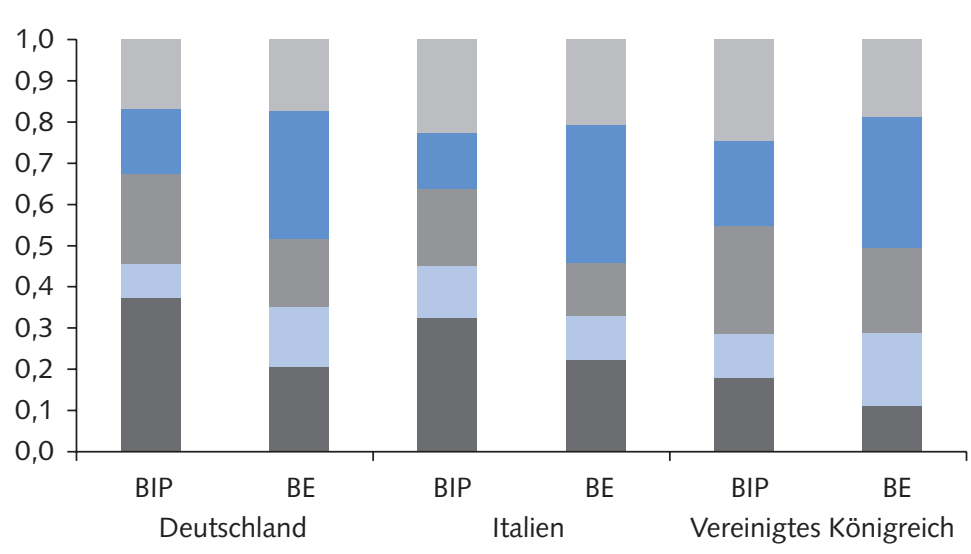

$$
\begin{aligned}
& \text { Andere } \\
& \text { Priv. u. Öffentl. Dienstleister } \\
& \text { Finanzen } \\
& \text { Handel } \\
& \text { Produzierendes Gewerbe }
\end{aligned}
$$

Anmerkungen: Die Kategorie „Finanzen“ beinhaltet Versicherungen, Immobilien und Business Dienstleistungen. Die Kategorie "Andere" umfasst Landwirtschaft, Bergbau, Energieversorgung, Baugewerbe, Hotels und Restaurants, Verkehr, Lagerhaltung und Kommunikation.

Quelle: GDDC KLEMS Datenbank, Berechnungen der Autoren.

WSI MITTEILUNGEN

fällig ist dabei die starke Spezialisierung in nur drei Kategorien von Gütern (Autos und Autoteile, Maschinen sowie chemische Produkte), die $200846 \%$ aller deutschen Exporte ausgemacht haben (Gehle-Dechant et al. 2010, S. 15). Im letzten Aufschwung $^{2}$ nahm die Bruttowertschöpfung im Verarbeitenden Gewerbe um $18 \% \mathrm{zu}$, in den Bereichen „Finanzierung, Vermietung und Unternehmensdienstleistungen“ und „Handel, Transport und Kommunikationsdienstleistungen" dagegen nur um 13 \% bzw. 10 \% (Statistisches Bundesamt 2010a, eigene Berechnungen). Der starken Exportorientierung steht eine schwache Binnennachfrage gegenüber. So stieg in den beiden Branchen „Baugewerbe“ und „öffentliche und private Dienstleister" die Bruttowertschöpfung im gleichen Zeitraum nur um jeweils $6 \%$.

\subsection{WER WAR VON DER KRISE BETROFFEN?}

Ein erstes Anzeichen der Krise war im 2. Quartal 2008 der Rückgang des Bruttoinlandsprodukts. Ebenso gingen die Exporte deutlich zurück und es kam zu einem Einbruch der Nachfrage nach Kraftfahrzeugen und Maschinen. Ab dem 4. Quartal intensivierte sich der Rückgang und belief sich im Jahresdurchschnitt 2008 auf 14,5 \%. Dies war der erste Rückgang seit 1992, wobei es in Deutschland zuvor keinen vergleichbaren starken Einbruch gegeben hatte. Ursächlich hierfür war, dass die Exporte von Gütern um 16,5 \% einbrachen, während die Exporte von Dienstleistungen lediglich um 1,9\% abnahmen (Statistisches Bundesamt 2010b). Wegen seiner hohen Exportabhängigkeit war das Verarbeitende Gewerbe besonders stark betroffen. Vier Quartale nach dem Beginn der Krise war die Bruttowertschöpfung im Verarbeitenden Gewerbe um mehr als $20 \%$ zurückgegangen (Statistisches Bundesamt 2010a, eigene Berechnungen). Im Gegensatz hierzu war die Situation im Dienstleistungsbereich und in der Banken- und Versicherungswirtschaft wesentlich besser. So waren laut einer Umfrage des Instituts für Arbeitsmarkt- und Berufsforschung (IAB) im 2. Quartal 2009 in diesen beiden Bereichen nur $20 \%$ bzw. $30 \%$ der Betriebe ganz oder teilweise von der Wirtschaftskrise betroffen, während $70 \%$ aller Betriebe in der Metallindustrie von der Krise betroffen waren (Möller 2010, S. 332).

Dies bestätigen auch die Ergebnisse einer Studie von Bogedan et al. (2009) auf Grundlage der WSI-Betriebsrätebefragung 2009. Demnach sind Betriebe in Westdeutschland, Betriebe mit einer

2 In Logeay/Zwiener (2008) beginnt der Aufschwung mit dem 4. Quartal 2004 und endet mit dem 1. Quartal 2008. 
starken Exportorientierung und Betriebe aus den Bereichen „Grundstoffe und Produktionsgüter" und „Investitions- und Gebrauchsgüter" stärker von der Krise betroffen als Betriebe in Ostdeutschland oder aus dem Dienstleistungsbereich. Diese Differenzierung spiegelt sich auch in den Beschäftigungseffekten wider (vgl. Rosemann/Kirchmann in diesem Heft). So sind Männer, Beschäftigte in exportorientierten Unternehmen, in Süddeutschland, Beschäftigte in Vollzeit und ohne abgeschlossene Berufsausbildung häufiger von Arbeitslosigkeit betroffen als Frauen, ältere Beschäftigte, Beschäftigte in Teilzeit oder in Minijobs.

Von der Wirtschaftskrise wurden hauptsächlich starke Firmen in wirtschaftlich starken Regionen getroffen, die vor der Krise die stärkste Nachfrage nach Fachkräften hatten (Möller 2010, S. 330f.). Es sind hauptsächlich die Firmen, die das Wachstum in Deutschland vor der Krise angetrieben haben und über Fachkräftemangel geklagt haben. Sie schaffen das „Deutsche Arbeitsmarktwunder" (Krugman 2009), weil sie finanziell in der Lage sind, Arbeitskräfte zu horten und sich wegen der Erfahrungen vorangegangener Krisen freiwillig dafür entschieden haben, ihre qualifizierten Belegschaften zu behalten, um sie sofort wieder zur Verfügung zu haben, wenn die Nachfrage erneut anzieht.

\subsection{MASSNAHMEN ZUR ABFEDERUNG DER KRISE}

Zur Abfederung der Krise hat die Bundesregierung neben dem Bankenrettungspaket eine Reihe von Maßnahmen zur Stützung der Konjunktur beschlossen, die in den sogenannten Konjunkturpaketen 1 und 2 und dem Wachstumsbeschleunigungsgesetz gebündelt auf den Weg gebracht wurden. ${ }^{3}$ Diese Konjunkturprogramme umfassen eine Vielzahl von Maßnahmen, die in drei verschiedenen Kategorien zusammengefasst werden können: Maßnahmen zur Beschäftigungssicherung, Maßnahmen zur Schaffung von Arbeitsplätzen und andere Maßnahmen.

Zur Krisenbewältigung hat Deutschland einen starken Schwerpunkt auf Maßnahmen zur Beschäftigungssicherung gelegt. Dazu gehören unter anderem die Ausweitung der bestehenden Regelungen zur Kurzarbeit, um die Nutzung von Kurzarbeit zu fördern und den Unternehmen finanzielle Anreize zu geben, an ihrer
Belegschaft in der Rezession festzuhalten (vgl. Bogedan in diesem Heft), sowie der Ausbau des Weiterbildungsprogramms „Weiterbildung Geringqualifizierter und beschäftigter älterer Arbeitnehmer in Unternehmen“" (WeGebAU) für ältere und gering qualifizierte Arbeitnehmer und eine dauerhafte Herabsenkung der Beiträge zur Arbeitslosenversicherung von 3,3\% auf $3 \%$ ab 2009, die befristet von Anfang 2009 bis Juli 2010 sogar auf nur 2,8 \% gesenkt wurden. Daneben gibt es eine Anzahl direkter und indirekter Hilfen für Unternehmen. Der Schwerpunkt der beiden Konjunkturpakete lag auf der indirekten Hilfe für Unternehmen, wozu als bekannteste Maßnahme die Abwrackprämie zählt, durch die der Einbruch in der Automobilbranche deutlich verschoben wurde. Die Programme umfassen aber auch staatliche Investitionen in die Infrastruktur, Investitionen in die Gebäudesanierung, zusätzliche Finanzmittel zur Verbesserung der regionalen Wirtschaftsstruktur und für strukturschwache Kommunen, aber auch eine befristete Kfz-Steuerbefreiung von neu zugelassenen Pkws oder die bessere Absetzbarkeit von Handwerksleistungen. Im Gegensatz dazu lag der Schwerpunkt des Wachstumsbeschleunigungsgesetzes auf der direkten Unterstützung von Unternehmen, wie z. B. die äußerst umstrittene Absenkung der Mehrwertsteuer für Beherbergungsleistungen.

Weit weniger Maßnahmen wurden zur Schaffung von neuen Arbeitsplätzen ergriffen. Im Wesentlichen beschränkten sich diese auf die Schaffung von 5.000 neuen Vermittlerstellen bei den Arbeitsagenturen. Ebenso spielten andere Maßnahmen in den Konjunkturpaketen eine untergeordnete Rolle. Zu nennen wäre hier eine Einmalzahlung je Kind von $100 €$, die Erhöhung des Kindergelds, des Kinderfreibetrags und der Regelsätze der 6- bis 13-jährigen Kinder in der Grundsicherung für Arbeitsuchende.

\subsection{SITUATION IN DER KRISE}

Für Deutschland war die globale Wirtschaftskrise die größte Krise in der Nachkriegszeit. Herzog-Stein/Seifert (2010) zeigen anhand eines Zyklenvergleichs aller Rezessionen seit den 1970er Jahren, dass es vier Quartale nach Beginn der Rezession in keiner anderen Krise einen derartigen Einbruch des Bruttoinlandproduktes $(6,9 \%)$ und der Arbeitsstunden (3,3\%) gab. Ein Jahr nach der Krise ist die registrierte Arbeitslosigkeit - im Gegensatz zur historischen Erfahrung und zu fast allen anderen Ländern in der EU - nicht angestiegen, sondern sogar um 1,3\% gefallen. Und zum jetzigen Zeitpunkt gibt es keine Anzeichen dafür, dass noch ein großer Anstieg der Arbeitslosigkeit zu befürchten wäre. Für diese positive Entwicklung am Arbeitsmarkt sind verschiedene Faktoren verantwortlich:

(1) Der Strukturwandel im Zusammenspiel mit der Begrenzung der Krise auf einen Teilbereich der deutschen Wirtschaft: Der Abbau der Beschäftigung von Vollzeitstellen im Verarbeitenden Gewerbe wurde durch die Schaffung von Teilzeitstellen im Dienstleitungsbereich mehr als kompensiert (vgl. Rosemann/Kirchmann in diesem Heft), allerdings nur quantitativ.

(2) Ein weit wichtigerer Grund war die Stabilisierung der Beschäftigung durch die Anpassung der Arbeitsstunden. Dieses wurde durch Maßnahmen der internen Flexibilität ermöglicht: Kurzarbeit, Abbau von Überstunden, Arbeitszeitkonten, spezielle Betriebsvereinbarungen, usw.

Wie groß der Einfluss von Maßnahmen der internen Flexibilität ist, wird in Studien wie unter anderem von Herzog-Stein/ Seifert (in diesem Heft) geschätzt. Sie kommen zu dem Schluss, dass ohne den Rückgang in der Arbeitszeit die Arbeitslosigkeit um 1,1 Mio. zugenommen hätte. Einen höheren Einfluss sehen sie im Horten von Arbeit, das zu einem Rückgang in der Arbeitsproduktivität geführt hat und rund 2 Mio. Jobs in der Krise gesichert hat. Die Bedeutung der Kurzarbeit ist in den Zahlen der Bundesagentur für Arbeit zu sehen. Es gab eine starke Zunahme der Kurzarbeit, die im Mai 2009 mit 1.533 Mio. registrierten Kurzarbeitern ihren Höhepunkt erreichte. Eine Auswertung der WSI-Betriebsrätebefragung (Bogedan et al. 2009) vom September 2009 zeigt, dass die Kurzarbeit von $20 \%$ der von der Krise betroffenen Betriebe genutzt wurde. Allerdings, werden Arbeitzeitkonten mit $30 \%$ noch weit häufiger genutzt. Dieses hat damit zu tun, dass vor allem das Verarbeitende Gewerbe von der Krise betroffen war und

3 Für Details siehe die zahlreichen Publikationen des IMK zu diesem Thema wie beispielsweise IMKArbeitskreis Finanzkrise (2009). 
dort Arbeitskonten häufiger verwendet werden. ${ }^{4}$ Die Betriebsrätebefragung zeigt darüber hinaus, dass Firmen zur Beschäftigungssicherung noch auf eine ganze Reihe anderer Maßnahmen in der Krise zurückgegriffen haben. Sie reichen von betrieblicher Umsetzung oder Versetzung von Mitarbeitern, Regelungen zur Urlaubsnahme, sonstigen Veränderungen in der Arbeitszeit bis hin zu Abstrichen beim Entgelt und Einschnitten bei den betrieblichen Sozialleistungen (vgl. Bogedan et al. 2009, S. 5).

\subsection{DAS DEUTSCHE ARBEITSMARKT- WUNDER}

Die Entwicklung auf dem Deutschen Arbeitsmarkt ist einmalig und kontrastiert mit allen bisherigen Erfahrungen: Trotz massivem Einbruch der Konjunktur hat es keine Massenentlassungen gegeben. Wie kam es zu diesem „Jobwunder“?

(1) Es gab eine klar erkennbare Strategie in den Maßnahmen gegen die Krise. Der Fokus lag eindeutig auf der Beschäftigungssicherung. Dazu hat in erster Linie die Ausweitung der Kurzarbeiterregelung beigetragen (vgl. Bogedan in diesem Heft), aber auch die Abwrackprämie, die bereits in einem frühen Stadium der Krise schnell und zielgerichtet den Einbruch der Automobilindustrie verzögert hat.

(2) Es lag aber auch daran, dass die Rahmenbedingungen äußerst günstig waren, sodass die Maßnahmen wirken konnten. Die Unternehmen, die von der Krise betroffen waren, waren finanziell in der Lage, die Kurzarbeiterregelungen in Anspruch zu nehmen, und wollten freiwillig an ihrer Arbeitsbelegschaft festhalten, was in vergangenen Krisen nicht der Fall war.

(3) In Deutschland haben die Sozialpartner - insbesondere in den von der Krise betroffenen Branchen - im Laufe der Zeit in einem verlässlichen Rahmen vertrauensvoll eine Vielfalt an Instrumenten der internen Flexibilität geschaffen, die es ermöglichen, den Arbeitseinsatz durch die Variation der Arbeitszeit an die Nachfragesituation anzupassen. Es lagen somit alle Merkmale, wie ein ausgeprägtes System von regulierter Flexibilität und Tarifvereinbarungen im Hinblick auf Arbeitszeiten und strikte Kündigungsschutzregelungen, vor, die laut OECD (2010a, S. 264) die Anpassung der Arbeitszeit fördern.
(4) Gleichzeitig trug der fortgesetzte Strukturwandel im Aggregat zur bemerkenswerten Arbeitsmarktentwicklung bei. Der Beschäftigungsaufbau von Teilzeitstellen im Dienstleistungsbereich verdeckt den Verlust von Vollzeitstellen im Verarbeitenden Gewerbe.

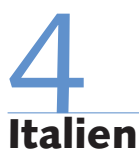

\subsection{VOR DER KRISE}

Der Schwerpunkt der italienischen Wirtschaft liegt in den Bereichen Lebensmittel, Landwirtschaft, Metall- und Anlagebau, Textil- und Bekleidungsindustrie, Industriedesign und der Produktion von Einrichtungen und Ausstattungen. Dabei ist die Produktionsstruktur der Wirtschaft in „,industrielle Sektoren“ gegliedert, die geografischen Regionen entsprechen. Jeder Distrikt besteht aus einer großen Anzahl von kleinen und mittelgroßen Unternehmen, die bestimmte Aufgaben im Produktionsprozess übernehmen. Vor der Krise hat sich der Arbeitsmarkt gut entwickelt. Nach Angaben von Eurostat fiel die Arbeitslosenquote kontinuierlich von $11,2 \%$ (1995) auf 6,1\% (2007). Dabei ist der italienische Arbeitsmarkt sehr heterogen, insbesondere gibt es ein ausgeprägtes Nord-Süd-Gefälle. In den letzten Jahren hat sich der Arbeitsmarkt in seiner Struktur spürbar verändert. So gab es, wie auch in Deutschland, eine kontinuierliche Zunahme der atypischen Beschäftigung.

\subsection{WER WAR VON DER KRISE BETROFFEN?}

Italien wurde erst verzögert von der Krise getroffen, was vor allem daran lag, dass das italienische Bankensystem, im Gegensatz zu dem des VK und Deutschlands, kaum von der globalen Finanzkrise betroffen war, da es strengere Bilanzierungsregelungen hat. Die ersten ernsten Anzeichen der Krise wurden im letzten Quartal 2008 sichtbar, als das Bruttoinlandsprodukt um $1,9 \%$ einbrach. Dabei gingen die Exporte um 7,4\%, Investitionen um 8,9\% und der Konsum der privaten Haushalte um $2 \%$ zurück (Busalacci et al. 2009). Obwohl die italienischen Banken nicht von der Finanzkrise tangiert waren, wurde im Verlauf der Krise der Zugang zu Krediten für kleine und mittlere Unternehmen, die das Herz des italienischen Produzierenden Gewerbes sind, schwieriger. Der Automobilsektor und die Bauwirtschaft waren am stärksten von der Krise betroffen. Bei den Beschäftigten traf es vor allem diejenigen mit befristeten oder projektbezogenen Arbeitsverträgen und Leiharbeiter; in den Branchen traf es das Verarbeitende Gewerbe, die Textilindustrie, den Finanzsektor und die Unternehmensdienstleistungen.

\subsection{MASSNAHMEN ZUR ABFEDERUNG DER KRISE}

Italien hat, ebenso wie Deutschland, einen starken Schwerpunkt auf die Beschäftigungssicherung gelegt. Die italienische Variante des Kurzarbeitergeldes ist ein Lohnergänzungsfonds, der vom „Nationalen Institut für Soziale Fürsorge“ (INPS - Istituto Nazionale Previdenza Sociale) verwaltet und hauptsächlich vom Staat finanziert wird. Die Aufgabe des Fonds ist es, Betriebe zu unterstützen, die in wirtschaftliche Notlagen kommen, sodass Entlassungen oder Kurzarbeit drohen. Italien hat zur Abfederung des Schocks auf die zwei altbewährten Instrumente, die von diesem Fonds gespeist werden, zurückgegriffen: erstens Unterstützung von vorübergehenden Entlassungen ${ }^{5}$ bzw. Kurzarbeit (Cassa Integrazione Guadeagni, kurz CIG) und zweitens Unterstützung von Kurzarbeit durch sogenannte Solidaritätsverträge (Contratti di solidarietà).

Die CIG wurde in der Vergangenheit hauptsächlich von mittleren und großen Unternehmen im Produzierenden Gewerbe und Bausektor verwendet. In der Krise wurde die CIG auf eine größere Anzahl an Beschäftigten wie atypisch Beschäftigte und Auszubildende und eine größere Zahl von Betrieben ausgeweitet. Dabei werden zwei Programme unterschieden:

- Im Fall von Betrieben, die während einer Wirtschaftskrise ohne eigenes Ver-

4 Die Forschung zeigt, dass Arbeitzeitkonten in größeren Unternehmen, in Unternehmen mit Betriebsräten und abhängig von dere Höhe des eingesetzten Humankapitals häufiger verwendet werden (Seifert 2004, S. 3). Im Jahr 2007 hatten 53,7\% der Beschäftigten im Verarbeitenden Gewerbe Arbeitzeitkonten, während nur $47 \%$ der Beschäftigten im Dienstleistungssektor ein Arbeitszeitkonto hatten (Groß/Schwarz 2009).

5 Diese Beschäftigten werden in der Statistik nicht als arbeitslos erfasst. 
schulden in eine Notlage kommen, greift das normale Programm (CIGO). Hier ersetzt der Lohnergänzungsfonds $80 \%$ des Lohnausfalls, der entweder durch eine vorübergehende Entlassung oder Kurzarbeit entstanden ist, für eine maximale Dauer von 13 Wochen. Unter außergewöhnlichen Umständen kann die Zahlung der Lohnersatzleistung auf bis zu 52 Wochen verlängert werden.

- Das Programm CIGS ist speziell für Betriebe gedacht, die in wirtschaftlichen Schwierigkeiten sind. Es stellt finanzielle Hilfen für die Umstrukturierung oder Abwicklung bereit. In diesem Fall werden ebenfalls $80 \%$ des Lohnausfalls durch den Fonds ersetzt, aber für eine Länge von maximal 24 Monaten.

Zudem wurde in der Krise ein paralleles System von Fonds eingeführt, in das Beiträge vom Arbeitsministerium fließen. Es unterstützt kleine Unternehmen und Handwerksbetriebe, die keine Ansprüche am CIGO oder CIGS haben.

Eine weitere Maßnahme, die vom Lohnergänzungsfonds bezuschusst wird und darauf abzielt, Massenentlassungen zu verhindern, sind Solidaritätsverträge, die zwischen Arbeitgebern und Arbeitnehmern geschlossen werden. Hierbei werden Arbeitszeit und Entgelte aller Beschäftigten gleichermaßen reduziert; und der Lohnergänzungsfonds bezahlt Zuschüsse für ausgefallene Arbeitsstunden. In der aktuellen Krise wurden diese befristet für die Jahre 2009 und 2010 um 20 \% in Höhe von 80 \% des Lohnausfalls erhöht. Die maximale Dauer ist 24 Monate. Sie kann um weitere 24 Monate verlängert werden, im Süden Italiens sogar um 36 Monate.

Parallel dazu gab es eine Anzahl von Maßnahmen zur direkten Unterstützung von Unternehmen. So z.B. Kredite mit niedrigen Zinsen für ökologische Produkte, Verschiebung von Steuerzahlungen oder einen erleichterten Zugang zu Krediten für kleinere und mittlere Unternehmen, indirekte Hilfen für Unternehmen im Bereich der Schlüsselindustrien wie elektrische Geräte, Einrichtungen und Ausstattungen, Verarbeitendes Gewerbe und Automobilindustrie (Abwrackprämie).

Im Bereich Maßnahmen zur Schaffung von Arbeitsplätzen wurden unter anderem ein neuer Fonds, der die öffentliche Bereitstellung von Bildungsressourcen erweitern soll, und ein Fonds, der Beschäftigung und Jungunternehmer unterstützt, eingeführt.
Zudem wurden Anreize geschaffen, um Empfänger von krisenbedingten Lohnersatzleistungen in die Selbstständigkeit zu führen, und die Arbeitsagenturen wurden besser vernetzt.

Weitere Maßnahmen umfassen die Verlängerung des Arbeitslosengeldes bis zu einem Maximum von 90 Tagen $^{6}$ für alle Beschäftigten, die keinen Anspruch auf eine Unterstützung durch den CIGFonds haben, eine Einmalzahlung für Leiharbeiter oder Beschäftigte, die bei Beendigung eines Projektvertrags arbeitslos wurden, und Unterstützungszahlungen für Haushalte und Kinder. Zudem wurden Übergangshilfen für Ladenbesitzer und Journalisten eingeführt, die kurz vor ihrer Verrentung stehen und ihre Berufstätigkeit aufgeben. Die Banken, die sich in einer Umstrukturierung befanden, riefen ein spezielles Frühverrentungssystem für ihre Beschäftigten ins Leben, die arbeitslos wurden und weniger als fünf Jahre vor der Rente standen. Sie erhalten $70 \%$ von ihrem Bruttolohn bis zu ihrer Verrentung.

\subsection{SITUATION IN DER KRISE}

Die Lage am Arbeitsmarkt hat sich im Verlauf der Krise stark verschlechtert. Der Einsatz von Kurzarbeit konnte einen kräftigen Anstieg der Arbeitslosigkeit bereits in der ersten Jahreshälfte 2009 zwar verhindern, aber mit dem Auslaufen dieser Maßnahmen gab es einen spürbaren Anstieg der Arbeitslosenquote nach dem Sommer 2009. So lag die Quote im Februar 2010 bei $8,5 \%$, was immer noch niedriger als im Euroraum insgesamt ist (vgl. PG 2010, S. 29), jedoch stieg die Arbeitslosigkeit im letzten Jahr um 1,2 \% an, was oberhalb des OECD-Durchschnitts lag (vgl. OECD 2010c). Auch andere Arbeitsmarkt-Indikatoren deuten einen eher pessimistischen Ausblick für die Entwicklung des italienischen Arbeitsmarkts an. So ist der Anteil der Beschäftigten an der arbeitsfähigen Bevölkerung von einem sowieso schon niedrigen Niveau seit dem letzten Quartal 2007 um 1,7 \% auf 57,1 \% gefallen. Allerdings zeigen die Zahlen der OECD auch, dass ohne die Reduzierung des Arbeitsvolumens um 2,7 \% die Beschäftigung noch weiter zurückgegangen und die Arbeitslosigkeit weiter angestiegen wäre.

\subsection{BRÜCKE INS NICHTS?}

Im Großen und Ganzen kann man sagen, dass Italien auf große Konjunkturpakete verzichtet hat, da Italien schon vor der Krise einen sehr hohen Verschuldungsstand hatte. Das Hauptaugenmerk zur Bekämpfung der Krise lag eindeutig auf der Beschäftigungssicherung durch Kurzarbeit und vorübergehenden Entlassungen (CIG). Die CIG gibt es bereits seit mehr als 60 Jahren in Italien und in jeder Krise wurde sie ein wenig ausgeweitet oder angepasst. Ursprünglich war die CIG dazu gedacht, die Einkommen der Beschäftigten in Betrieben während Krisenzeiten zu sichern, indem permanente Jobverluste durch befristete Entlassungen oder Kurzarbeit verhindert wurden. Allerdings zeigt die Erfahrung, dass sie immer häufiger auch in Fällen verwendet wird, in denen eine Rückkehr zur normalen Arbeitssituation äußerst unwahrscheinlich erscheint. Daher können die Zahlungen der CIG nicht länger als Maßnahmen zur Beschäftigungssicherung angesehen werden, sondern entsprechen eher einer Art Arbeitslosenunterstützung (vgl. Aricò/Stein 2010). Diese Entwicklung wurde sicher dadurch verstärkt, dass es in Italien keine Arbeitslosenversicherung so wie in Deutschland gibt.

Die Auswertung der Maßnahmen zur Beschäftigungssicherung zeigt, dass Italien noch nicht über den Berg ist. Zum aktuellen Zeitpunkt ist unklar, ob die Maßnahmen ausgereicht haben, denn alle wichtigen Kennzahlen steigen weiter an. So nimmt die Zahl der Beschäftigten, die Lohnersatzleistungen vom Lohnergänzungsfonds bekommen, weiter zu und hat Ende 2009 einen Höhepunkt mit fast $4 \%$ aller Beschäftigten in Kurzarbeit erreicht. Auch im 1. Quartal 2010 sind die Kurzarbeiterzahlen noch fast genauso hoch. Des Weiteren haben Anreize zur Frühverrentung, die wieder eingesetzt wurden, sicher mit dazu beigetragen, dass der sowieso schon niedrige Anteil der Beschäftigten an der Bevölkerung im arbeitsfähigen Alter wieder deutlich zurückgegangen ist. Diese Entwicklung wirft die Frage auf, ob fehlende Konjunkturprogramme die Ursache sind. Es besteht nämlich die Gefahr, dass in nächster Zeit, wenn die Berechtigung für das Kurzarbeitergeld ausläuft, viele Menschen noch ihren Job verlieren werden

6 In Italien gibt es kein breit angelegtes und einheitliches System der Arbeitslosenunterstützung. 
und das Instrument der CIG nicht mehr in der Lage sein wird, einen Anstieg der Arbeitslosigkeit zu verhindern (vgl. OECD 2010c). In diesem Fall wäre die Brücke zur Beschäftigungssicherung zu kurz gewesen. Die ergriffenen Maßnahmen hätten nicht ausgereicht, die krisenbedingten Probleme auf dem Arbeitsmarkt zu lösen; sie hätten sie lediglich verzögert.

\section{Vereinigtes Königreich (VK)}

\subsection{VOR DER KRISE}

Die Wirtschaft des VK erlebte eine lange Periode der Prosperität. Nach Angaben von Eurostat stieg zwischen 1992 und 2007 das Bruttoinlandsprodukt im Durchschnitt jährlich um rund 3,3\%. Die Arbeitslosenquote fiel im gleichen Zeitraum um 4,5 Prozentpunkte auf 5,4\%. Insbesondere der britische Finanz- und Bankensektor profitierte von der ökonomischen Globalisierung. So lagen 2006 der Export der Finanzdienstleistungen bei $2 \%$ und die Wertschöpfung der Kredit- und Versicherungswirtschaft bei $8 \%$ des Bruttoinlandprodukts (OECD 2009). Jeder Fünfte arbeitete in der Finanzbranche (Abbildung 1). Diese wirtschaftliche Entwicklung entpuppte sich im Nachhinein als nicht nachhaltig. Ein Hauptbestandteil war die Spirale von vereinfachten Zugängen zu Krediten, die daraus resultierende Zunahme von Wertpapierpreisen, die dann wieder neue Möglichkeiten boten, höhere Kredite abzusichern. Dies spiegelt sich auch in der Entwicklung des Immobilienmarkts wider. Die Immobilienpreise stiegen bis in die zweite Hälfte 2007 stark an. Zudem war die Sparquote des VK sehr niedrig. Der britische Konsum wurde hauptsächlich durch die Verschuldung der privaten Haushalte finanziert. Nach Angaben von Eurostat lag die durchschnittliche Verschuldung der privaten Haushalte ausgedrückt als prozentualer Anteil des verfügbaren Einkommens für 2006 bis 2008 bei $151 \%$, während sie in Deutschland und Italien nur bei 93 \% bzw. 56 \% lag.

\subsection{WER WAR VON DER KRISE BETROFFEN?}

Der starke Anstieg der Immobilienpreise vor der Krise zusammen mit der enormen
Abhängigkeit vom Finanzsektor hat das VK anfällig für die Krise gemacht, und so war das erste deutliche Anzeichen der Krise auch der starke Rückgang der Immobilienpreise. Britische Banken waren massiv von der globalen Finanzkrise betroffen und mehrere Institute wurden zeitweise teilverstaatlicht. Neben der Finanzbranche litt aber vor allem der Exportsektor, trotz starker Abwertung des Britischen Pfunds, unter massiven Einbrüchen.

Die Arbeitslosenquote stieg von 2007 bis 2009 um 2,3 Prozentpunkte auf 7,5 \%. Besonders von der Krise betroffen sind die unter 25-Jährigen und die gering Qualifizierten. Allein 2009 stieg die Arbeitslosenquote dieser Gruppen um $8 \%$ bzw. $12 \%$ (vgl. OECD 2010d). Bereits seit Beginn der 2000er Jahre ist die Jugendarbeitslosigkeit im VK gegen den OECD-Trend gestiegen und war schon 2008 mit $14 \%$ sehr hoch (Bell/Blanchflower 2009).

\subsection{MASSNAHMEN ZUR ABFEDERUNG DER KRISE}

Im Gegensatz zu Deutschland und Italien hat das VK nur wenige Maßnahmen zur Beschäftigungssicherung implementiert. Eine davon war die Erweiterung des bestehenden Weiterbildungsprogramms („Train to Gain“) für Kurzzeitqualifikationen von Januar 2009 an. Es dient dazu, das Qualifikationsniveau von Beschäftigten zu verbessern. Nicht zuletzt aufgrund des langen Aufschwungs hatte das VK eine nur wenig ausgeprägte Infrastruktur der aktiven Arbeitsmarktpolitik (AAMP), auf die man nun in der Krise hätte zurückgreifen können. Weitere Maßnahmen wie die direkte Unterstützung von Unternehmen, die in die Produktion von grünen Produkten involviert sind, und die indirekte Unterstützung von Unternehmen in Form einer Abwrackprämie wurden umgesetzt. Mit der befristeten Absenkung der Mehrwertsteuer von 17,5\% auf $15 \%$ von Ende 2008 bis Ende 2009 sollte kurzfristig die Binnennachfrage angekurbelt werden.

Ein größerer Schwerpunkt wurde auf Maßnahmen zur Schaffung von Arbeitsplätzen für die unter 25-Jährigen und die Langzeitarbeitslosen in Regionen mit hoher Arbeitslosigkeit gelegt. Im Oktober 2009 wurde ein eine Milliarde Pfund teures Programm „Future Jobs Fund“ eingeführt, mit dessen Hilfe 150.000 neue Jobs geschaffen werden sollen, 100.000 davon für junge Erwachsene und 50.000 in Gebieten mit hoher Arbeitslosigkeit. Allerdings wurde dieses Programm von der neuen Regierung im Zuge des Sparhaushalts bereits wieder gestoppt. Des Weiteren wurde die Anzahl der Vermittlerstellen um knapp $5 \%$ aufgestockt. Da die Arbeitsuchenden im Zeitraum der Krise aber um 75 \% zugenommen haben, ist dies sehr wenig (OECD 2010d). Zusätzlich wurde für Langzeitarbeitslose, die bereit sind, an einer Weiterbildung teilzunehmen, ein Zuschlag auf die Arbeitslosenunterstützung gezahlt. Arbeitgeber, die einen Langzeitarbeitslosen eingestellt haben, bekamen einen Zuschuss. Ebenso werden alternative Formen der Beschäftigung gefördert, wie die Schaffung von Jobs in sozialen und nicht gewinnorientierten Organisationen, die Übernahme von Teilbürgschaften für Kredite, um sozialorientierte Unternehmen in benachteiligten Gebieten zu fördern, und die Unterstützung von Arbeitslosen beim Übergang in die Selbstständigkeit.

\subsection{GERINGE FINANZMITTEL, UNKLARE STRATEGIE}

Bewertet man die britischen Maßnahmen zur Krisenbewältigung zusammenfassend, fällt es schwer, eine klare Strategie zur Krisenbekämpfung festzustellen. Dieses liegt sicher daran, dass das VK durch drei Kanäle von der Krise betroffen war. Durch die enorme Abhängigkeit von der Finanz- und Bankenbranche waren enorme Anstrengungen notwendig, um das Bankensystem zu stützen. Dies hat die Spielräume bei der Bekämpfung des Nachfrageschocks durch die globale Rezession deutlich eingeschränkt. Die Mittel, die notwendig wären, um die Konjunktur weiter anzukurbeln bzw. den Anstieg der Arbeitslosigkeit zu bekämpfen, fehlen. So wirken viele der Maßnahmen nur wie ein Tropfen auf den heißen Stein. Ebenso fehlen spürbare Impulse durch AAMP. Was bleibt, sind die Versuche, die Arbeitslosigkeit der Problemgruppen mit Sonderprogrammen in Schach zu halten. Die Zukunftsaussichten für die Entwicklung des VK sind sehr unsicher. Nachdem im Mai 2010 eine neue konservativ-liberale Regierung ins Amt gewählt wurde und diese dem Land in aller

\footnotetext{
7 Nicht nur die Staatsverschuldung, sondern auch die Verschuldung der privaten Haushalte spielt eine Rolle, um die drohende Überschuldung eines Landes zu beurteilen (vgl. Horn et al. 2010).
} 
Eile einen radikalen Sparkurs ${ }^{8}$ verordnet hat, warnen viele, „dass das Blut-Schweißund-Tränen-Programm, das mehr als eine Million Arbeitsplätze kosten könnte, das Land zurück in die Rezession zu werfen drohe“" (Theurer 2010). Zudem ist dem Nothaushalt der neuen Regierung gleich das Programm „Future Jobs Funds“ - eines der wenigen zur AAMP - zum Opfer gefallen. Weitere Sparmaßnahmen sehen große Ausgabenkürzungen in den Ministerien vor. Es ist inzwischen sogar die Rede davon, die Beschäftigung im Öffentlichen Dienst abzubauen. Gegeben, dass diese Personengruppe bisher auf Grundlage einer sicheren Beschäftigung Zukunftspläne, inklusive Wohneigentum, gemacht hat, würde eine Entlassungswelle hier sicher eine Reihe weiterer schwerwiegender Folgen nach sich ziehen, insbesondere durch die große Bedeutung des kreditfinanzierten Immobilienkaufs im VK. Der kreditfinanzierte Immobilienkauf hat im VK eine sehr viel größere Bedeutung als in Deutschland.

\section{Diskussion und Vergleich der Länder}

Durch die größere wirtschaftliche Integration des VK waren die Auswirkungen der globalen Finanzkrise deutlich stärker als in Deutschland und Italien. Britische Banken waren besonders stark von der Immobilienkrise betroffen. Deutschland war zwar auch von der Bankenkrise betroffen, hat es aber durch schnelles eigenes Eingreifen und aufgrund der amerikanischen Rettungsmaßnahmen geschafft, die Bankenkrise relativ glimpflich zu überstehen. Italien hingegen war gar nicht von einer Bankenkrise, dafür aber hart von der globalen Rezession betroffen.

Wie Leschke/Watt (2010) zeigen, kann die AAMP als kurzfristiger Puffer zur Verhinderung eines schnellen Anstiegs der Arbeitslosigkeit verwendet werden. Die unterschiedliche Wirkungsweise der AAMP kann man am Beispiel der hier analysierten Länder erkennen; die scheinbar gleichen Maßnahmen zur Kurzarbeit sind besonders interessant.

Das VK, das durch einen über viele Jahre anhaltenden Aufschwung einen hohen Beschäftigungsstand und eine niedrige Arbeitslosigkeit hatte und von vielen für seine enorme externe Flexibilität als Vorbild angesehen wurde, hat in der Krise nun einen starken Einbruch erlebt. Es ist nicht gelungen den Anstieg der Arbeitslosigkeit zu begrenzen, vor allem nicht bei den Problemgruppen der Jüngeren und Geringqualifizierten, für die es bereits vor der Krise spezielle Programme gab. Die Ausgaben und die Beteiligung von Menschen vor der Krise an der AAMP waren sehr gering, und so blieb es auch nach Beginn der Krise. Der Fokus der Maßnahmen liegt im VK auf der Vermittlung von Arbeitslosen und nicht auf Maßnahmen, die einer längerfristigeren Perspektive bedürfen. Erst spät wurde mit Aktivierungsmaßnahmen reagiert, also erst nachdem die Arbeitslosigkeit schon deutlich angestiegen war. Zudem hatte das VK für diese Art der Maßnahmen auch nur noch wenig Ressourcen zur Verfügung.

Italien andererseits kann mit dem seit mehr als 60 Jahren existierenden Lohnergänzungsfonds auf eine lange Tradition der AAMP zurückgreifen. Wie Deutschland hat Italien mit Kurzarbeit reagiert. Anders als in Deutschland besteht in Italien aber die Gefahr, dass es keine Erfolgsgeschichte wird, sondern der Anstieg der Arbeitslosigkeit nur hinausgeschoben wurde. Der Hauptgrund liegt wohl darin, dass der Lohnergänzungsfonds zwar bei jeder Krise angepasst wurde, aber er sich immer mehr von einer Maßnahme zur Beschäftigungssicherung zu einem System der Arbeitslosenunterstützung gewandelt hat. Im Gegensatz zu Deutschland werden viele Kurzarbeiter nämlich zeitweise entlassen, sodass sie zwar arbeitslos sind, aber nicht in der Arbeitslosenstatistik gezählt werden. Während die Arbeitslosigkeit in Italien im ersten Jahr der Krise im EUVergleich nur unterdurchschnittlich anstieg, ist sie im zweiten Jahr der Krise überdurchschnittlich gestiegen. Zusätzlich ist anzumerken, dass (während in Deutschland der Anteil der Beschäftigten, die in Kurzarbeit waren, bereits im 3. Quartal 2009 deutlich zurückging) die Anzahl der italienischen Kurzarbeiter bis Ende 2009 weiter deutlich anstieg und seither auf hohem Niveau verharrt. Dieses lässt aber befürchten, dass in nächster Zeit, wenn die Berechtigung für das Kurzarbeitergeld ausläuft, viele Beschäftigte noch ihren Job verlieren werden und der CIG nicht mehr in der Lage sein wird, das Steigen der Arbeitslosigkeit weiter zu verhindern (OECD 2010c).
Auch in Deutschland stellt sich die Situation völlig anders dar als im VK. Es gab schon vor der Krise einen starken Fokus auf die AAMP. In der Krise wurde schnell reagiert und es hat sich gezeigt, dass die Maßnahmen der internen Flexibilität, die sich im Laufe der Zeit immer weiter entwickelt haben, enorm mit dazu beigetragen haben, das Arbeitsvolumen über die Stunden, und nicht über die Köpfe anzupassen. Aktuell spricht vieles dafür, dass Deutschland ohne eine große Zunahme der Arbeitslosigkeit aus der Krise kommen wird. Woran liegt das? In Deutschland wurde die bestehende Kurzarbeiterregelung schnell an die Krisenerfordernisse angepasst. Allerdings hat die Kurzarbeit trotz großer Effekte in der aktuellen Krise nur einen Teil zur Beschäftigungssicherung beigetragen. Ebenso haben in Deutschland die Instrumente der internen Flexibilität (Arbeitzeitkonten, Abbau von Überstunden, Anpassung der wöchentlichen Arbeitszeit, etc.), die sich in den letzten Jahren weiterentwickelt haben, dazu beigetragen. Diese Instrumente der internen Flexibilität konnten sich entwickeln, weil es staatliche Regelungen gibt, die es den Sozialpartnern ermöglichen, in einem verlässlichen Rahmen miteinander $\mathrm{zu}$ verhandeln. In der letzten Krise hat sich nun gezeigt, wie wertvoll das Zusammenspiel der vielfältigen Instrumente der internen Flexibilität ist.

\section{Fazit}

Die vorangegangen Fallbeispiele haben illustriert, wie und warum die Länder unterschiedliche Wege in der Krisenbewältigung gegangen sind. Für das VK fällt es dabei schwer, eine klare Strategie auszumachen. Dieses liegt aber daran, dass das VK gleich durch mehrere Kanäle von der Krise ge-

8 Der Nothaushalt von Georg Osborne für 2010 sieht Ausgabenkürzungen in Höhe von $40 \mathrm{Mrd}$. $£$ vor. Dazu gehören massive Ausgabenkürzungen von bis zu $25 \%$ im Öffentlichen Dienst, starke Einschnitte bei den Sozialausgaben wie die Kürzung bzw. Abschaffung von einer Reihe von Beihilfen für Familien mit Kindern bei gleichzeitiger Anhebung der Mehrwertsteuer auf $20 \%$ und vieles mehr. Dieses ist nur ein erster Sparplan, der in aller Eile direkt nach den Wahlen von den Konservativen und Liberalen aufgesetzt wurde. Es ist die Rede davon, dass das, was danach kommt, sogar die rigorosen Sparpläne der Thatcherjahre übertreffen wird. 
troffen wurde und somit schon gleich zu Beginn mit enormen Mitteln den Finanzund Bankensektor stützen musste, was die Mittel für andere Krisenmaßnahmen reduziert hat. Außerdem liegt der Fokus im VK traditionell auf der Vermittlung von Arbeitslosen und der Schaffung von Arbeitsplätzen. Deswegen spielt die AAMP, im Gegensatz zu Deutschland und Italien, die eine lange Tradition der AAMP haben, auch nur eine untergeordnete Rolle. Italien hat wegen seiner hohen Staatsverschuldung auf große Konjunkturpakete verzichtet und dabei auf Altbewährtes gesetzt. Es ist eine klare Strategie zur Beschäftigungssicherung durch eine AAMP zu erkennen, die ganz auf den Einsatz von Kurzarbeit orientiert. Hier bleibt allerdings noch abzuwarten, ob die Brücke, die dieses Instrument gebildet hat, ausreicht, um über die Krise hinweg zu tragen, oder ob dieses Mittel zu kurz greift. Gegeben, dass den Betrieben keine anderen Mittel der internen Flexibilität, die die Kurzarbeit ergänzt hätten, zur Verfügung standen, ist zu befürchten, dass die Kurzarbeit, anstatt den
Anstieg der Arbeitslosigkeit zu begrenzen, diese nur zeitlich verschoben hat. Ähnlich wie Italien hat auch Deutschland eine klar erkennbare Strategie zur Beschäftigungssicherung durch Kurzarbeit gewählt. Anders als in Italien stand den Betrieben aber eine Vielzahl von Instrumenten der internen Flexibilität zur Verfügung, die die Betriebe ergänzend zur Kurzarbeit nutzen konnten. Gerade dieses Zusammenspiel scheint nun in der Krise der entscheidende Unterschied zwischen Erfolg und Misserfolg zu sein.

\section{LITERATUR}

Aricò, F./Stein, U. (2010): Employment and economic recession in Germany, Italy, and UK: different remedies for the same illness?, unveröffentlichtes Manuskript

Bell, D./Blanchflower, D. (2009): What Should Be Done About Rising Unemployment in the UK?, IZA Discussion Paper 4040

Bogedan, C./Brehmer, W./Herzog-Stein, A. (2009): Betriebliche Beschäftigungssicherung in der Krise, WSI-Report 01

Busalacchi, J./Calzolari, G./Chiurco, F./D'Alessandro, L./D'Alessio, A./de Mottoni, G./Di Saverio, M./Fasano, G./Ferri, P./Guzzo, I./ Mangano, G./Moretti, S./Mosca, M./Racioppo, A. (2009): Risposte europee all'attuale crisi socio-economica (Dossier Paesi), ISFOL and FSE, Ministero del Lavoro e delle Politiche Sociali, Italien

Gehle-Dechant, S./Steinfelder, J./Wirsing, M. (2010): Export, Import Globalisierung - Deutscher Außenhandel und Welthandel, 1990 bis 2008, Statistisches Bundesamt, Wiesbaden

Groß, H./Schwarz, M. (2009): Arbeitszeit, Altersstrukturen und Corporate Social Responsibility, Wiesbaden

Herzog-Stein, A./Seifert, H. (2010): Stabile Beschäftigung durch flexible Arbeitszeiten, in: Arbeit - Zeitschrift für Arbeitsforschung, Arbeitsgestaltung und Arbeitspolitik (im Erscheinen)

Horn, G./Niechoj, T./Tober, S./van Treeck, T./Truger, A. (2010): Reform des Stabilitäts- und Wachstumspakts: Nicht nur öffentliche, auch private Verschuldung zählt, IMK-Report 51

IMK-Arbeitskreis Finanzkrise (2009): Von der Finanzkrise zur Wirtschaftskrise (I) - Wie die Krise entstand und wie sie überwunden werden kann, IMK-Report 38

Krugman, P. (2009): Germany's jobs miracle, in: New York Times 17.09. Leschke, J./Watt, A. (2010): How do institutions affect the labour market adjustment to the economic crisis in different EU countries?, European Trade Union Institute (ETUI), Working Paper 04
Logeay, C./Zwiener, R. (2008): Deutsche Realeinkommensverluste für Arbeitnehmer: Die neue Dimension eines Aufschwungs, in: WSI-Mitteilungen 8, S. 415-421

Möller, J. (2010), The German labor market response in the world recession - de-mystifying a miracle, in: Zeitschrift für ArbeitsmarktForschung 4, S. 325-336

Organisation für wirtschaftliche Zusammenarbeit und Entwicklung (OECD) (2009): Economic Surveys - United Kingdom 9

Organisation für wirtschaftliche Zusammenarbeit und Entwicklung (OECD) (2010a): Economic Outlook 87

Organisation für wirtschaftliche Zusammenarbeit und Entwicklung (OECD) (2010b): Employment Outlook - Moving Beyond the Job Crisis Organisation für wirtschaftliche Zusammenarbeit und Entwicklung (OECD) (2010c): Länderreport Italien

Organisation für wirtschaftliche Zusammenarbeit und Entwicklung (OECD) (2010d): Länderreport Vereinigtes Königreich

Projektgruppe Gemeinschaftsdiagnose (PG) (2010): Erholung setzt sich fort - Risiken bleiben groß - Gemeinschaftsdiagnose Frühjahr 2010, IMK-Report 47

Seifert, H. (2004): Flexibility through working time accounts: reconciling economic efficiency and individual time requirements, WSI Discussion Paper 130

Statistisches Bundesamt (2010a): Volkswirtschaftliche Gesamtrechnung -Inlandsproduktberechnung, Vierteljahresergebnisse, 1. Vierteljahr 2010, Fachserie 18, Reihe 1.3, Mai, Wiesbaden

Statistisches Bundesamt (2010b): Volkswirtschaftliche Gesamtrechnung -Inlandsproduktberechnung, Vierteljahresergebnisse, 1. Vierteljahr 2010, Fachserie 18, Reihe 1.2, Mai, Wiesbaden

Theurer, M. (2010): David Cameron ist Mister Thatcher, www.faz.net, 27. August 